\title{
Blood Vessel Segmentation and Centerline Extraction based on Multilayered Thresholding in CT images
}

\author{
Shagufta*, Shoab.A.Khan ${ }^{\dagger}$, A.Hassan ${ }^{\ddagger}$ and S. Rashid ${ }^{\ddagger}$ \\ *College of Electrical and Mechanical Engineering National University of Sciences and Technology, \\ Islamabad,44000,Pakistan \\ ${ }^{\dagger}$ College of Electrical and Mechanical Engineering National University of Sciences and Technology, \\ Islamabad,44000,Pakistan \\ ${ }^{\ddagger}$ College of Electrical and Mechanical Engineering National University of Sciences and Technology, \\ Islamabad,44000,Pakistan
}

*Email: shagufta.bena@gmail.com

\begin{abstract}
Information retrieval from Coronary Computed Tomography Angiography (CCTA) is the most frequently used tool by physicians to diagnose cardiac diseases. However, the use of Coronary Computed Tomography Angiography in this context is quite rare. We have introduced a novel approach to detect severity of cardiac diseases using CCTA images. The proposed technique consists of multiple steps algorithm which extracts blood vessels from CCTA images of patients to analyze disease symptoms. The major steps are: Pre-processing, Multilayered thresholding and centerline extraction. The proposed technique has been tested on dataset containing fifty images collected from different hospitals located in different cities. The results obtained are highly encouraging; we could achieve $92 \%$ accuracy.
\end{abstract}

Keywords-Coronary Computed Tomography Angiography (CCTA), Cardiac Disease, blood vessels, centerline extraction.

\section{INTRODUCTION}

Accurate visualization of the human vasculature is an important feature for a number of clinical settings. In the image application fields medical imaging becomes inevitability for physicians, especially at the times of diagnosing the cardiovascular diseases. Coronary Computed Tomography Angiography (CCTA) images have significant role in detection and diagnosis of many cardiac diseases for cardiologist. With the development of technology, non-invasive imaging modalities have become more attractive and frequently used in clinical procedures. One of its applications is Coronary Computed Tomography Angiography (CCTA) which is most widely used for patients at present. CTA has been used in several clinical applications includes cardiac, neck and head, abdomen. CCTA is better at revealing the calcified plaques and blood vessels, both having high intensity in image. CCTA is used to view the coronary arteries in order to analyze the blood flow in them. These arteries are those that supply blood to the heart muscle. Powerful X-ray machines generate images of heart and its vessels. Through these images, blockages in the arteries and narrowing of the vessels can be checked.In Figure.1 arrows showing the narrowing of the vessels.

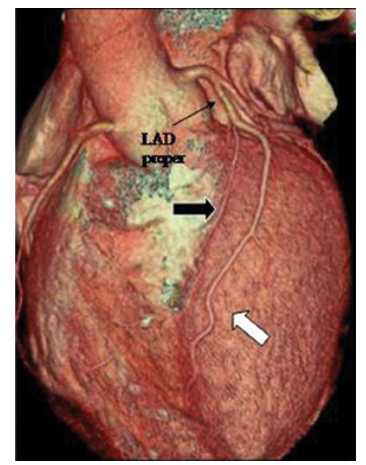

Fig. 1: CT angiography image indicating blocked artery.

A technique based on pattern recognition used in vessel segmentation is proposed by Wang Shou-jue et al. ${ }^{(1)}$, it was experimented with the Biometric Pattern Recognition by using artificial neural networks that act through covering the geometrical distribution of high dimensional sample set in the feature space ${ }^{(2-3)}$. N. Orlov et al. ${ }^{(4)}$, proposed a method, in the first-stage Image pyramids, sub-band filters, and image transforms were used ${ }^{(5)}$. Fourier wavelet, chebyshev transforms can also recognize the patterns ${ }^{(6-7)}$. Qiang wu ${ }^{(8)}$ applied the wavelet-based image enhancement technique to a well-known biomedical pattern recognition problem. Most studies were aimed to improve the quality of display of image for visualization, by enhancing the visibility of low contrast image features while suppressing noise. The criteria for success differ among various methods but are usually related to the local image contrast measure and/or 
contrast improvement ratio ${ }^{(9-10)}$. Classical thresholding variations have been proposed that combined information based on connectivity and local intensities. A survey of thresholding techniques can be found in ${ }^{(11)}$. A. Frangi et al. (12) proposed to detect vesselness using the eigen-values of Hessian matrix that distinguishes the line-like structure from blob-like and plate-like structures and suppressed random noise present in the background. Chuadhuri et al. (13) presented a method that used the matched filter to emphasize the vessels. The region based thresholding of response of the matched filter method was proposed by Hoover. ${ }^{(14)}$. Soares et al. ${ }^{(15)}$ proposed a technique using the 2D Gabor wavelet to segment the blood vessels. M. Akram (16) proposed automated vessel segmentation technique using 2D Gabor wavelets and multi-layered thresholding. This paper presents the blood vessels enhancement and segmentation method for Coronary CT Angiography images. Blood vessels segmentation is not an easy task because of the thinness of the blood vessel and low contrast between the background and edges of the vessel. The proposed technique, first enhance blood vessel patterns by using the contrast enhancement, morphological operator and 2D Gabor wavelet filter and then apply a multi-layered thresholding method. The organization of this paper consists of four parts. Section I provides an introduction to Coronary Computed tomography Angiography before reviewing the existing blood vessels enhancement and segmentation techniques in the literature. Section II explains the proposed method. Section III includes the experiments and results. Conclusion and the possible future direction are presented in the section IV.

\section{Methodology}

To develop the automated vessel enhancement and segmentation system, the initial step is to collect the dataset from an extensive collection of raw data of CCTA scan. Dataset was collected from various hospitals, espacially Armed Forces Institute of Cardiology (AFIC) and Leading Reading Hospital (LRH) Peshawar. We created two data-sets, one is named as AFIC data-set and other is LRH data-set. The automated blood vessel segmentation system facilitate the cardiologists is an application of medical system. Figure. 2 is the block diagram of the proposed technique to automated blood vessels enhancement and segmentation from CCTA images.

\subsection{Pre-Processing}

The CCTA images are mostly poorly illuminated and noisy due to the camera motion and unknown noise. Due to these factors the visibility of blood vessel is not good. So, first enhance the vessels pattern.

\subsubsection{Conversion from Rgb to Gray scale}

The input RGB image is converted into gray image as shown in Figure. 3(c).

\subsubsection{Contrast Enhancement}

The noisy images first need to be cleaned by using some pre-processing steps including the contrast enhancement technique, morphological operator and also to remove noise from image we applied the median filter. The enhanced image replaces each pixel value by:

$$
I(j, k) \leq c * I(j, k)+(1-c) * \operatorname{avg}(j, k)
$$

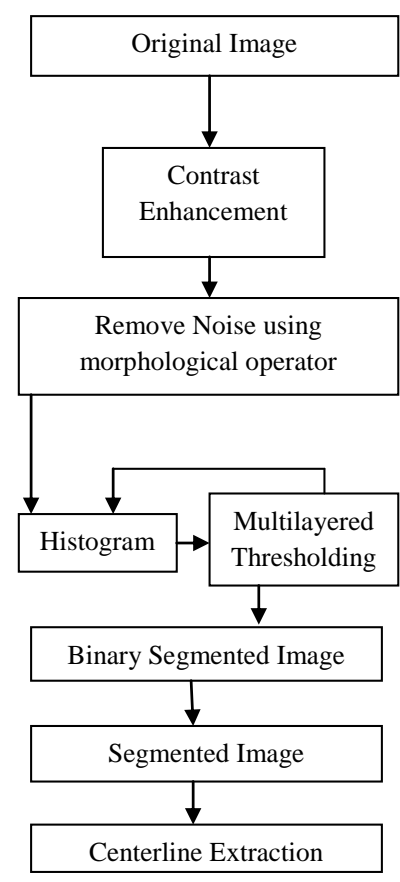

Fig. 2: Block diagram of the proposed technique.

In Equation 1, 'c' is the contrast parameter. Contrast between blood vessels and image background is increased by using contrast enhancement technique. Then performed the morphological top-hat filtering on the enhanced grayscale image to get the more enhanced image. Median filter is applied on the enhanced image that remove the noise from the background. Pre-processing results are shown in Figure. 3

\subsection{Create a binary mask}

After the enhancement of blood vessels, morphological operation and multi-layered threshold methods are used to create a binary mask for blood vessels segmentation. The blood vessels are extracted by using the technique of masking that assign 1 to the blood vessel pixels and 0 to nonvessel pixel. For blood vessels segmentation, multi-layered thresholding technique takes 2D- Gabor wavelet response as input. Thick blood vessels have large values of $I_{\text {wavelet }}$ whereas $I_{\text {wavelet }}$ has low values where the blood vessels are thin. From the figure it is clear that at center vessel have high response and low response for thin blood vessels and on edge pixels. Gabor wavelet has directional selective criteria. They perform as low level oriented edge discriminators; removed the noise from background of image and identifying the oriented features and fine adjusting to particular frequencies (17). Gabor wavelet response is shown in Figure. 4.

The 2D continuous wavelet transform $F \varphi(i, \theta, j)$ is the scalar product of $\mathrm{j}(\mathrm{x}), \mathrm{f}(\mathrm{x}, \mathrm{y})$ with the transformed wavelet using (2)

$$
F \psi(i, \theta, j)=A \psi^{-1 / 2} i^{-1} \int \psi_{i, \theta, j}^{*}(x) f(x, y) d^{2} x
$$

Where $x=[x y]^{T}, f \psi \Re^{2}$ is image represented as an integrable function, which is defined over $\Re^{2}$ and $\psi$ analyzing wavelet. $\psi^{*}$ represents the complex conjugate of 


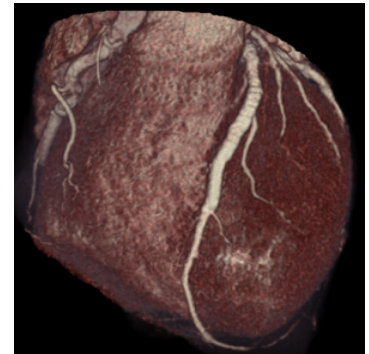

(a) Original image

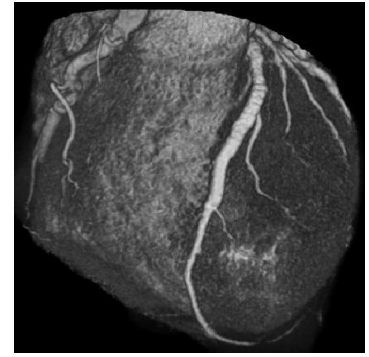

(c) Gray scale image

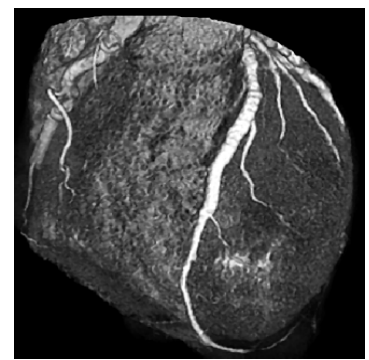

(e) After the morphological operator and median filter

Fig. 3: Pre-processing steps: (a) Original colored image (b) RGB enhanced image (c) Gray image (d) Gray scale enhanced image (e) Final image after noise removal

TABLE 1: Parameters for Gabor wavelet

\begin{tabular}{|l|c|}
\hline Factors & Value \\
\hline Dilation(a) & 3.7 \\
Elongation $(\epsilon)$ & 4 \\
Rotation angle $(\theta)$ & $15^{\circ}$ \\
$\mathrm{K}_{o}$ & {$\left[\begin{array}{ll}0 & 3\end{array}\right]$} \\
\hline
\end{tabular}

$\psi . A \psi$, , i', 'j' and $\theta$ represent the constant of normalizing, the vector of displacement, dilation parameters and the angle of rotation respectively.

\subsection{Segmentation}

Binary segmented image shown in Figure. 5 is used to extract the blood vessels from gray scale image. The final segmented image contain vessel with their original intensities.

\subsection{Centerline Extraction of blood vessels}

Several techniques have been proposed for extraction of skeleton of blood vessels. In literature most of the methods are based on distance transform ${ }^{(18-19)}$. In this paper, we

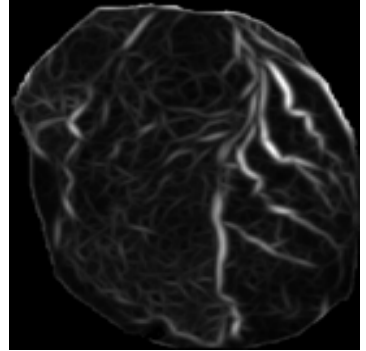

(a)

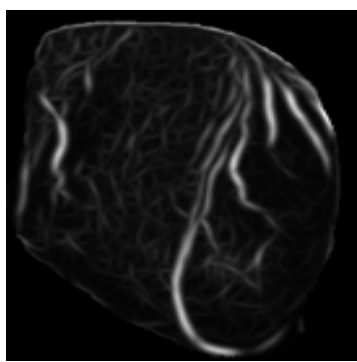

(b)
Fig. 4: Gabor wavelet response

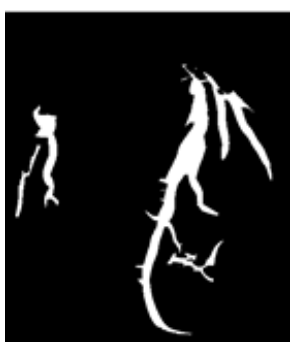

Fig. 5: Binary mask.

used method that is based on morphological operations, which performed erosion using $2 \times 2$ neighborhoods. If all four of the neighborhoods pixels are on only then the output pixel is on. Centerline extraction result is shown as figure. 6

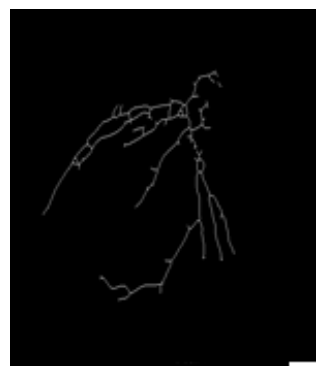

Fig. 6: Blood vessel centerline

\section{Results}

To measure the performance of this proposed technique, we have created the dataset that contains the normal patient CCTA images as well as abnormal images. The proposed method is done in MATLAB R2010b and run on a $2.5 \mathrm{GHz}$ Intel Core i5 Laptop with 8GB Ram. We test the different angles images on proposed algorithm. After segmentation, most of the detected region is the true blood vessels pixel but some of the regions are non-blood vessel pixels. Figure. 7 shows how the blood vessels detected.

The manual segmentation of images is performed by physicians that are used as actual blood vessels. Receiver operating characteristic (ROC) graph is used for the performance of proposed technique. ROC curve is a plot with the true positive rate versus false negative rate. Accuracy results 


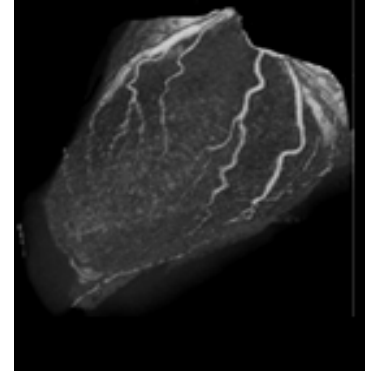

(a)

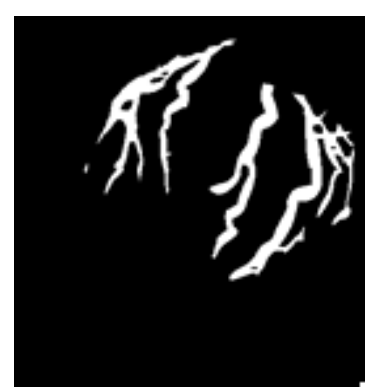

(c)

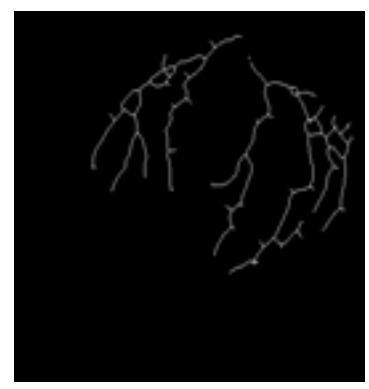

(e)

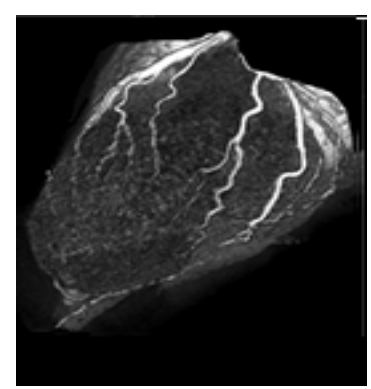

(b)

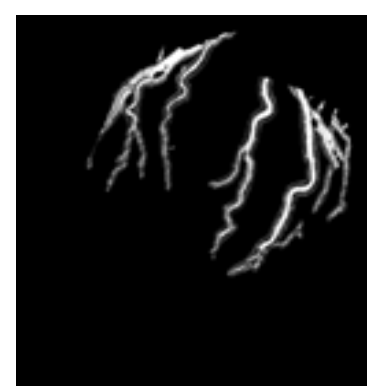

(d)

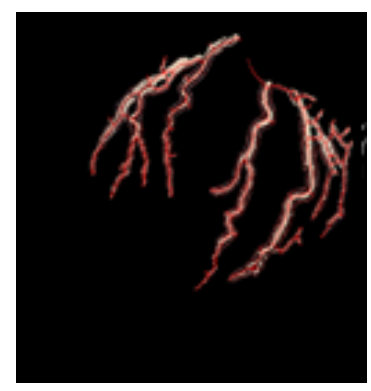

(f)
Fig. 7: (a) Original image (b) Enhanced image (c) Binary mask (d) Segmented image (e) centerline extraction (f) Merged the (d) and (e)

are calculated for AFIC and LRH data-sets. We calculated the accuracy using the following parameters.

TABLE 2: Parameters for Accuracy

\begin{tabular}{|l|l|l|l|}
\hline \multicolumn{2}{|c|}{} & \multicolumn{2}{|c|}{ Predicted } \\
\cline { 3 - 4 } \multicolumn{2}{|c|}{} & False & True \\
\hline \multirow{2}{*}{ Actual } & False & a & b \\
\cline { 2 - 4 } & True & c & d \\
\hline
\end{tabular}

True Positive (TP): Pixels, which are calculated as blood vessels pixels and also they belong to actual blood vessel pixels.

False Positive (FP): Pixels, which are calculated as blood vessel pixels but they are not actual blood vessel pixels.

True Negative (TN): Pixels, which are non-blood vessels and also they are non-blood vessel pixels in actual.

False Negative (FN): Pixels, which are calculated as nonblood vessels but they are actual blood vessel pixels.

True positive rate (TPR) is calculated by dividing TP pixels by the total number pixels that belong to blood vessels shown in equation (3). False positive rate (FPR) is fraction of false positive pixels and the total number of pixels that

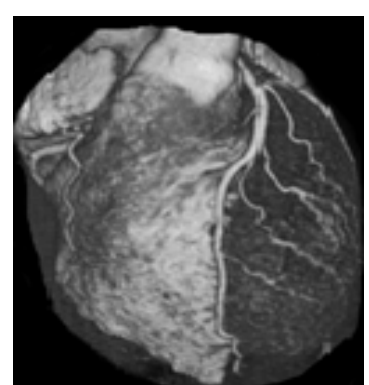

(a)

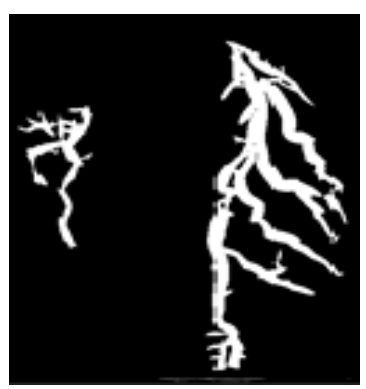

(c)

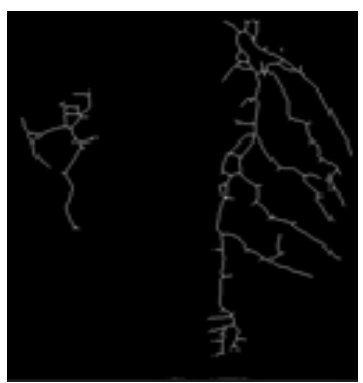

(e)

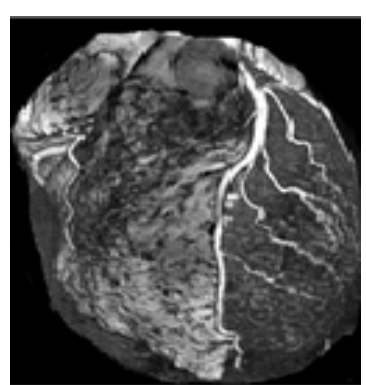

(b)

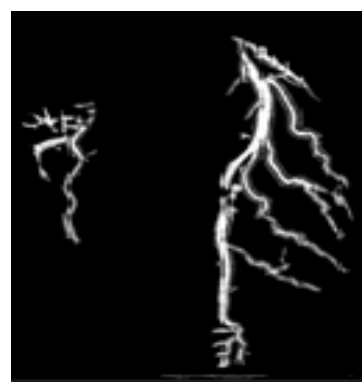

(d)

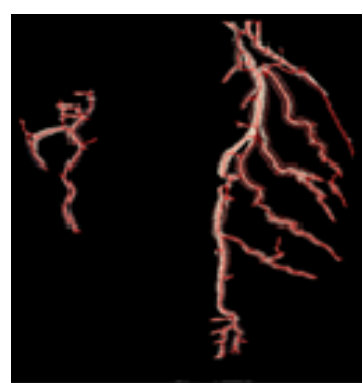

(f)
Fig. 8: (a) Original image (b) Enhanced image (c) Binary mask (d) Segmented image (e) centerline extraction (f) Merged the (d) and (e)

are non-blood vessel pixels given as equation (4). Figure .9 shows the ROC curves for AFIC and LRH data-sets produced by proposed method.

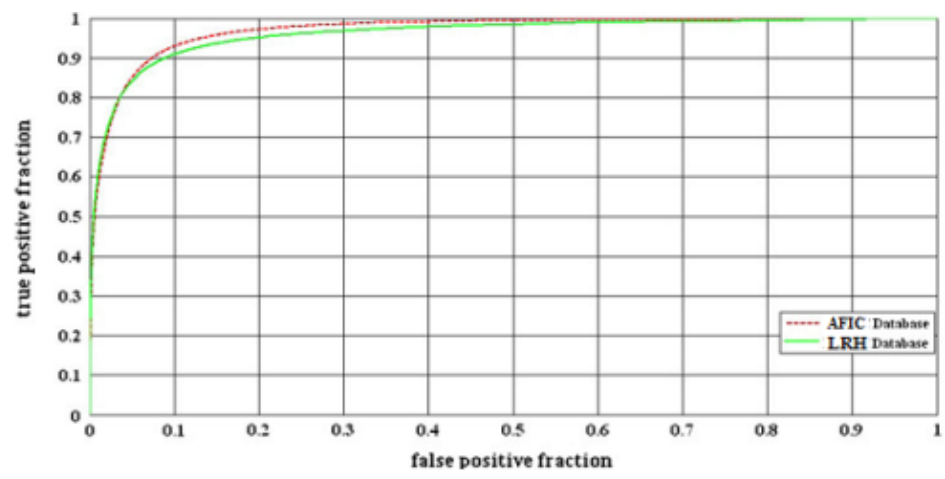

Fig. 9: ROC curves for AFIC and LRH data-sets

$$
T P R=\frac{d}{c+d}
$$




$$
\begin{gathered}
F P R=\frac{b}{a+b} \\
A=\frac{a+d}{a+b+c+d}
\end{gathered}
$$

TABLE 3: Accuracy results for vessel segmentation

\begin{tabular}{|l|l|}
\hline Database & Accuracy \\
\hline AFIC & 0.9482 \\
\hline LRH & 0.8957 \\
\hline Average & $\mathbf{0 . 9 2 1 9}$ \\
\hline
\end{tabular}

Table 3 shows the calculated accuracy values for both databases using the equation (5). These are averaged accuracy values.

\section{Conclusion}

In the diagnosis of coronary arteries diseases (CAD), segmentation of blood vessels has a significant role to play. In this paper, an algorithm is presented for the segmentation of blood vessels in CCTA images. The CCTA images subjected to pre-processing including the contrast enhancement and morphological techniques. The proposed methodology is based on multilayered thresholding and morphological techniques. The proposed method validation is executed quantitatively by comparing with manual segmentation of vessels. It can provide a convenient way for physicians to extract the beneficial information about the blood vessels.

\section{REFERENCES}

[1] Wang Shoujue, Analysis \& Theory of High-Dimension Space Geometry for ANN, J. Acta Electrronica Sinca, 30(1), pp. 1-4, 2002

[2] R. Fisher, Contributions to Mathematical Statistics, J. Biometrika, 38(1/2), pp. 257-259, 1951

[3] V.N,Chervonenkis. Moscow \& Vapnik, Theory of Pattern Recognition, 1974

[4] N. Orlov, Multipurpose image classification using compound image transform, J. Pattern Recognit Lett, 29(11), pp. 1684-1693, 2008
[5] K.Fukunada, Introduction to statistical pattern recognition, Academic Press, 1990

[6] P. Burt and E. Adelson, The Laplacian pyramid as compact image code,J. IEEE Trans. on Communication , 31(4), pp. 532-540, 1983

[7] N. Orlov, T. Macura, J. Johnston, I. Goldberg and C. Wolkow, Pattern recognition approaches to compute image similarities, application to age related morphological change, Biomedical Imaging: Nano to Macro , pp. 1152-1156, 2006

[8] Y.Wang, Z. Xiong and K. Castleman, Image enhancement using multiscale differential operator, Acoustics, Speech \& Signal Processing, 3, pp. 1853-1856, 2001

[9] Y.Wang, Image enhancement using multi-scale differential operator, Image Processing ,IEEE transaction, 8(12), pp. 1757-1771, 1999

[10] M.D'Zmura, K. Knoblauch and P. Colantoni, Color transparency,Perception , 26 , pp. 471-492, 1997

[11] P.K Sahoo, A.K Wong and S. Soltani, A Survey of Thresholding Techniques, J. Computer Vision, Graphics, \& Imag. Processing, 41(2), pp. 233-260, 1988

[12] A. Frangi, L. Vincken, M. Viergever and W. Niessen, Multi-scale Vessel Enhancement Filtering, Med. Image Computing \& ComputerAssisted Interventation, 1496 , pp. 130-137,1998

[13] S. Chaudhuri, M. Goldbaum, M. Nelson, N. Katz and S.Chatterjee, Detection of blood vessels in retinal images using two dimensional matched filters, IEEE Transaction Medical Imaging, 8(3), pp. 263269,1989

[14] A. Hoover, M. Goldbaum and V. Kouznetsova, Locating blood vessels in retinal images by piecewise threshold probing of matched filter response, IEEE Transaction Medical Imaging, 19(3),pp. 203-211, 2000

[15] J. Soares, M. Cree, H. Jelinek, R. Cesar and J. Leandro, Retinal vessel segmentation using the 2-D Gabor wavelet and supervised classification, IEEE Transaction Medical Imaging, 25(9), pp. 12141222, 2006

[16] M. Akram and S.A. Khan, Multi-layered thresholding based blood vessel segmentation for screeing of diabetic retinopathy, 29(2), pp. 165-173, 2013

[17] J. Antoine, B. Piette and P. Carette, Image analysis with 2D continuous wavelet transforms, Signal Processing, 31(3),pp. 241-272, 1993

[18] M. Maddah, A. Afzali-khusha and H. Soltanian and, Snake modeling \& distance transform approach to vascular center line extraction and quantification, J. Computerized Med. Imag. \& Graphics, 27 (6), pp. 503-512, 2003

[19] M. Wan, A. Kaufman , I. Bitter, L.Hang, and Z.Liang, Automatic Center line Extraction for Virtual Colonoscopy, IEEE Transaction Medical Imaging, 21(12), pp. 1451-1460, 2002 